\section{EL LENGUAJE DE LA CIENCIA}

\author{
César Lorenzano \\ Universidad Nacional de Tres de Febrero \\ Buenos Aires (Argentina)
}

\title{
THE LANGUAGE OF SCIENCE
}

\begin{abstract}
From the beginning of philosophy of science, neo positivism proposed physicalism as the basic, common language to all scientific disciplines. We all know of its difficulties and failures. I will analyze the language used in a case of applied -and also basic- research in clinical medicine, so as to evaluate if it is possible an unique language in science, or if it is an unreachable dream.

Even when our analysis is structuralist, this case allows us to reconstruct in a natural way the medical clinical theory by means of its exemplars, and not by mathematical models.

When we finish our analysis, it will be evident that the old dream of neo-positivism is carried out, and that the language of science-more precisely, the language suitable to reconstruct scientific theories- is a kind of physicalist - and also nominalist-one.
\end{abstract}

KEY WORDS: Language of science; reconstruction of theories; semiology; physicalism; nominalism; structuralist metatheory; exemplars; resemblance nominalism; infectious diseases; pneumonia.

\section{INTRODUCCIÓN}

Hacia los años veinte del siglo pasado varias corrientes teóricas se condensan en lo que se ha dado en llamar la "concepción heredada" de la filosofía de la ciencia, y que surge a la consideración pública como programa de investigación metacientífica en el "Manifiesto del Círculo de Viena" y en escritos posteriores. El neopositivismo hegemoniza el horizonte metateórico hasta por lo menos los años sesenta, cuando la obra de Thomas Kuhn introduce la necesidad de tomar en cuenta, y muy seriamente, los aportes de la historia de la ciencia a la hora de caracterizar el conocimiento científico.

Fundamentalmente las corrientes que convergen en el neopositivismo son las siguientes:
RESUMEN: En el comienzo de la filosofía de la ciencia, el neopositivismo propuso que el fisicalismo fuera el lenguaje básico, común a todas las disciplinas científicas. Sabemos de sus dificultades, y de sus fracasos.

En el presente artículo me propongo analizar el lenguaje empleado en un caso de investigación aplicada -y asimismo básica- perteneciente al campo de la clínica médica, a los efectos de sopesar la posibilidad de que exista un lenguaje único de la ciencia, o si éste es un sueño inalcanzable.

Aunque el análisis sea estructural, el caso posibilita de una manera natural la reconstrucción de la teoría clínica médica mediante la caracterización de sus ejemplares, prescindiendo, por lo tanto, de modelos matemáticos.

Al finalizar el recorrido, nos reencontramos con el viejo sueño neopositivista, al proponer que el lenguaje básico de la ciencia -en realidad, el lenguaje más apropiado para la reconstrucción de las teorias científicas- es de índole fisicalista y nominalista.

PALABRAS CLAVE: Lenguaje de la ciencia; reconstrucción de teorías; semiología; clínica médica; fisicalismo; nominalismo; concepción estructuralista; ejemplares; nominalismo de semejanzas; enfermedades infecciosas; neumonía.

i. El "giro lingüistico", considerado como una tendencia generalizada en la filosofía, por la cual el objeto de análisis deja de ser ideas o conceptos, para serlo el lenguaje, las expresiones lingüisticas, por considerarse -entre otros motivos- que constituyen aquello que es público y, por lo tanto, intersubjetivo.

ii. La nueva lógica de Frege, Russell y Whitehead, que brinda las herramientas para su análisis.

iii. El empirismo como teoría del conocimiento, que si bien tiene una larga historia, su más próximo antecedente es Ernst Mach, el científico, filósofo e historiador de la ciencia que antecedió a Moritz Schlick en la cátedra universitaria en cuyo seminario se fundó el Círculo de Viena.

iv. $Y$, casi nunca tomado en cuenta, el esperantismo, la utopía de un lenguaje común para toda la humanidad, 
que permitiera que los hombres pudieran finalmente entenderse, superando los obstáculos de la Babel lingüistica.

Quisiera mencionar por último el fuerte hálito nominalista que tiene toda la empresa neopositivista -reconocido explícitamente por Otto Neurath, y en menor medida por Rudolf Carnap-, y que se fue dejando de lado por la filosofía de la ciencia -a nuestro parecer innecesariamente-.

Es innecesario señalar que éstos son hitos alrededor de cuya elucidación se construyó gran parte de la filosofía de la ciencia contemporánea, sea para continuarlos, o para desarrollarlos bajo otros puntos de vista.

El presente artículo intenta contribuir a esta discusión, que involucra tanto al lenguaje de la ciencia y su estructura, como a su ontología y a las herramientas de análisis, tomando en cuenta los desarrollos de concepciones post neopositivistas tales como las de Thomas Kuhn y la concepción estructuralista de las teorías.

Comencemos, pues, con el relato de una investigación aplicada en el que aparece la totalidad del lenguaje de una teoría determinada, para proceder posteriormente a su análisis estructural, ontológico y epistemológico.

\section{El Lenguaje de una inVeStigación}

El Sr. K tuvo hace aproximadamente una semana un estado gripal invernal, del que se recupera lentamente. En el dia de la fecha amanece con fiebre, elevación de la frecuencia del pulso, moderada a severa dificultad respiratoria y una tos dolorosa de carácter paroxístico, con expectoración herrumbrosa o directamente sanguinolenta. Cuando concurre a la consulta médica, presenta dolor espontáneo en el tórax, que el Dr. M atribuye a la proximidad de la lesión interna de la enfermedad. Con sus dedos percute la zona, sintiendo cómo se produce un sonido mate -matidez-, en contraste con la sonoridad del resto del tórax. Cuando lo ausculta, oye en ese sitio un soplo tubárico, en vez de la suave crepitación y entrada de aire habituales. Al llegar a este punto, $\mathrm{M}$ formula la presunción de que se trata de una neumonía. La radiografía de tórax corrobora el diagnóstico, que es reafirmado cuando el análisis bacteriológico del esputo confirma la presencia del neumococo, agente habitual de la neumonía.

El Dr. M -y K, por consiguiente- son afortunados. El resto del examen clínico es normal, por lo que no incide en la evolución de la enfermedad. Tampoco presenta alteraciones tales como derrame pleural, o insuficiencia cardíaca provocadas por la misma enfermedad. K, que ha enfermado por acción del neumococo, curará después de algunos días de tratamiento. Antes de la era antibiótica, a la semana de iniciada la enfermedad curaba si tenía lugar un episodio que se denominaba crisis, en el que bajaba la fiebre y comenzaba la mejoría, o simplemente el paciente moría.

M sabe que ningún paciente es igual a otro -de la misma edad-, el mismo estado general previo -tanto orgánico como inmunológico-. Tampoco presenta la lesión pulmonar en el mismo lugar, ni la misma combinatoria de lesiones asociadas, así como tampoco evolucionan de la misma manera; no responde de igual forma al tratamiento con antibióticos, e incluso que pese a lo característico de la imagen radiológica, las lesiones pulmonares pueden deberse -si así lo evidencia el análisis bacteriológico del esputo- a otros microorganismos.

Sin embargo, puede diagnosticar y prever la evolución del paciente -su pronóstico-. Si analizamos el proceso por el cual infiere la índole de la enfermedad de $\mathrm{K}$, no hallamos rastros de que haya apelado a una deducción basada en una ley general de la neumonía -por otra parte, ausente en los libros de texto-.

¿Cómo lo hace? ¿En qué basa la seguridad con la que diagnostica y trata a $K$ ? ¿Cuál es el lenguaje, el léxico específico con el que describe y diagnostica la enfermedad?

En lo que sigue intentaremos responder a estas preguntas, centrales, como pienso, a la hora de comprender el lenguaje de la ciencia, sus elementos y su estructura, así como la forma inferencial que compromete.

Mi apuesta es sostener que nos encontramos ante un caso paradigmático de utilización de una teoría -que llamaré, a falta de mejor nombre- una teoría clínico-infecciosa, a la que se parecerán las demás teorías científicas cuando son utilizadas para caracterizar el mobiliario del mundo que recortan, y del que intentan predecir su comportamiento. 


\section{El ANÁlisis del LeNGUAJE}

Luego de narrar esta historia, que coincide, aunque simplificada, con la historia clínica de $K$, analizaré su estructura léxica. En ella el Dr. M deja constancia de sus hallazgos; encontramos las huellas de sus inferencias en los estudios complementarios que pide. La escribe para recordar los datos de $\mathrm{K}$ cuando lo vuelva a ver, o para que otros puedan constatarlos -controlando la exactitud de los mismos- 0 continúen tratando el paciente sin comenzar desde cero -si esto fuera necesario-. Por otra parte, la evolución que experimenta la enfermedad, y que queda asimismo registrada en la historia clínica es parte importante del diagnóstico y del pronóstico -si sigue la evolución prevista, y si ésta permite ser optimista o pesimista-. Recordemos que las historias clínicas son parte de la tradición médica iniciada por Hipócrates, quien nos deja en sus escritos el registro de más de cuarenta enfermedades diferentes.

Cuando iniciamos el análisis terminológico de la historia clínica, advertimos que describe lo que se denominan signos y sintomas del paciente: lo que puede observarse, y lo que cuenta el paciente. Palabras como fiebre, tos de determinadas características, percusión mate, soplo tubárico describen aquello que surge de la exploración del cuerpo de $\mathrm{K}$-los signos de la enfermedad-. Anotamos como sintomas la fatiga que experimenta -aunque también podemos percibirla-, el dolor torácico, la sensación de agotamiento y enfermedad.

Como constatamos, no se trata de un empirismo de sensaciones, como lo quería el primer neopositivismo, que pronto lo abandona dado el carácter subjetivo de las mismas, que por esta circunstancia mal podía ser el fundamento objetivo -intersubjetivo- del conocimiento científico. Si bien es cierto que son descripciones de fenómenos espacio-temporales -con lo que coincide con el fisicalismo, la segunda tentativa neopositivista de fundamentar el conocimiento- tampoco se superpone estrictamente con éste, al menos en sus versiones más estándares. Aunque se refiere a situaciones macroscópicas, se aleja notablemente de los objetos del conocimiento común, del conocimiento habitual.

Es mucho más próximo a un lenguaje básico que recién se describió como tal -viendo el fracaso de intentos anteriores- alrededor de principios de los años setenta, y que Hempel (1973) Ilama de conocimiento anterior, y la concepción estructuralista (Sneed, 1971) como no-teórico, proveniente de una teoria previa -precisando aún más la posición de Hempel-.

Desde el comienzo de la medicina, Hipócrates es consciente de estas distinciones. Los signos y sintomas no son recogidos al azar, ni obedecen a una observación común. Se inscriben en una disciplina especial -la semiología-, que teoriza acerca de signos y sintomas, enseñando a buscarlos, y por supuesto a verlos. La matidez de la percusión, o el soplo tubárico no pertenecen al lenguaje común, ni a la experiencia común. Se trata de un lenguaje especializado, en un campo teórico de la observación médica asimismo especializada. Un lenguaje y una experiencia provenientes de una teoría previa - presupuesta- a la teoría clínica de la neumonía, y que le brinda su "base empirica" -si nos permitimos usar una expresión ya casi perimida-.

En segundo lugar, a este lenguaje básico que describe lo que llamaremos la enfermedad semiológica se le añade otro, mediante el cual $\mathrm{M}$ hace hipótesis acerca de qué alteraciones del pulmón -y orgánicas en general- causan la sintomatología de K. Decir que $\mathrm{M}$ piensa que se trata de una neumonía, equivale a decir que piensa que en la zona de dolor torácico, donde capta la matidez y el soplo tubárico, el aire del pulmón está reemplazado por un exudado de determinadas características, determinando lo que llama condensación pulmonar, y que ésta es la causa de la enfermedad semiológica. Si lo consideramos en unión a los signos y síntomas, tenemos caracterizada lo que acostumbra llamarse la enfermedad clínica, en la que ambos planos -uno más próximo a la experiencia que el otro- evolucionan conjuntamente. Este lenguaje diferente al semiológico se refiere a zonas del organismo que no son perceptibles más o menos directamente, y a alteraciones que se describen con un lenguaje perteneciente a al menos dos disciplinas especializadas, la anatomía patológica y la fisiopatología; luego añadiremos la microbiología'.

Como podrá apreciarse, este nuevo lenguaje posee las características de lo que se suele denominar lenguaje teórico, ya que se trata de un plano introducido para explicar la enfermedad semiológica, y sólo es pertinente si interviene causalmente en una enfermedad clínica definida.

Hacemos notar que ambos planos -semiológico y anatomofisio-patológico- evolucionan conjuntamente siguiendo eta-

ARBOR Vol. 187I 747 enero-febrero [2011] 15-24 ISSN: 0210-1963

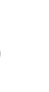


pas previstas -el pronóstico médico-, que es funcionalmente equivalente a la predicción en otras teorias científicas.

Finalmente, en una nueva vuelta de tuerca a la causalidad en medicina, en este caso especifico, al tratarse de una enfermedad infecciosa, se establece que es el neumococo el microorganismo que causa las alteraciones del pulmón, que a su vez causan los signos y síntomas de K. Si no hubiera microorganismo, no se trataría de una enfermedad infecciosa.

\section{La estructura del diagnóstico de K}

Partiendo de estas distinciones léxicas se podrá establecer la estructura del diagnóstico médico, cuyo caso paradigmático, en lo que hace a nuestras investigaciones epistemológicas, es la enfermedad neumónica de K.

Como observáramos, en el diagnóstico de $\mathrm{K}$ se siguieron tres etapas claramente diferenciadas:

i. La exploración de los signos y síntomas que permitieron encuadrarlo dentro de una enfermedad semiológica.

ii. La formulación de las hipótesis acerca de las alteraciones del pulmón, así como del agente causal microbiano.

iii. La corroboración posterior de las hipótesis previas, y de la evolución de la enfermedad -su pronóstico-.

Estas etapas corresponden, como es evidente, a tres caracterizaciones sucesivas de $\mathrm{K}$ que podemos denominar -siguiendo una terminología cercana a la de la concepción estructuralista-, no-teórica (semiológica), teórica (la hipótesis de que presuntamente presentará una condensación pulmonar), y finalmente actual ${ }^{2}$ (si se corroboran las hipótesis y se cumple la predicción -ley evolutiva- de la neumonía (su pronóstico).

Haremos notar que la ley evolutiva relaciona entre sí a todos los elementos que encontramos en la lectura de la historia clínica de $\mathrm{K}$, diciendo que signos, síntomas y lesiones evolucionan conjuntamente según se lo pronostica -hacia la curación o la muerte-. Se denomina a esta última caracterización actual, pues sólo si esto sucede, el paciente padece efectivamente -actualmente ${ }^{3}-$ una neumonía.

Las sucesivas etapas no han delimitado, como es habitual en la concepción estructuralista, a ningún modelo matemático, sino a un caso -en adelante un ejemplar- de la teoría microbiana de la neumonía.

Procederemos a continuación a mostrar mediante diagramas la estructura de ese ejemplar que se construye por etapas.

\section{K COMO EJEMPLAR NO-TEÓRICO}

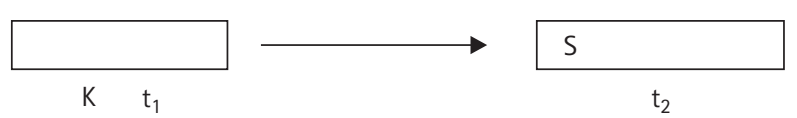

El diagrama se lee como sigue:

$K$ en el tiempo que va de $t_{1}$ a $t_{2}$ pasa de un estado previo -gripal, como recordaremos- a tener serios signos y sintomas.

El diagrama describe la enfermedad semiológica de $\mathrm{K}$ en sus aspectos más generales, sin especificar los signos y sintomas que posee, a fin de que sea apto -por su misma generalidad- para describir cualquier enfermedad semiológica, no sólo a $K$, y ni tan siquiera a la neumonía.

\section{K COMO EJEMPLAR teórico}

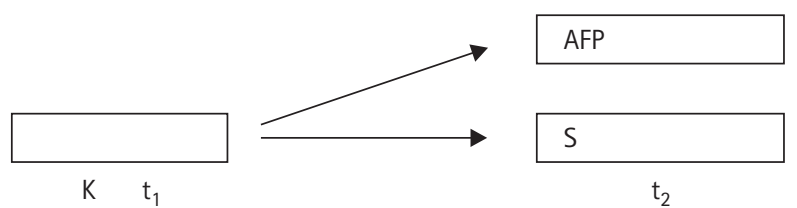

En esta descripción, $\mathrm{K}$-o un paciente cualquiera- no sólo tiene determinados signos y sintomas S. El Dr. M supone -hipotéticamente- que también presenta alteraciones anatomo-fisio-patológicas AFP intimamente relacionadas con S, en las que puede encontrarse el bacilo neumónico. Hechos que deberá corroborar mediante estudios específicos. 


\section{K COMO EJEMPLAR ACTUAL}

Para que K sea un ejemplar actual -efectivo- de una neumonía, deben cumplirse dos condiciones.

La primera de ellas, que se corroboren las alteraciones que supuso $M$, mediante estudios complementarios -en este caso, radiografías y análisis bacteriológicos-.

Esta condición da cuenta de la realidad actual de los elementos del ejemplar teórico.

Posteriormente, en lo que constituye la segunda condición, esos elementos deben evolucionar conjuntamente tal como lo especifica el pronóstico -su axioma evolutivo-. El axioma evolutivo relaciona entre sí todos los elementos que caracterizan a $\mathrm{K}$, uniendo indisolublemente los no teóricos con los teóricos. En ausencia de medios auxiliares de diagnóstico -antes de los rayos $X, 0$ en ambientes muy alejados de un centro hospitalario-, el cumplimiento de la evolución prevista, con su crisis que pone fin a la fiebre y al decaimiento a la semana de iniciado el proceso, y la desaparición de la matidez y el soplo tubárico un mes después, puede constituirse en una instancia legítima de corroboración del diagnóstico, ya que sólo la neumonía evoluciona de esa manera. El paso del tiempo, y por consiguiente de la sintomatología, es también en otras afecciones uno de los medios de corroboración de las hipótesis clínicas.

Si no evoluciona como se prevé, pudiera tratarse de otra enfermedad que simula una neumonía, y la presencia del microorganismo algo totalmente accesorio, dado que el neumococo es una habitante habitual del organismo, que ocasionalmente causa la enfermedad a la que da nombre.

Ahora sí, si se cumplen ambas condiciones $\mathrm{K}$ es efectivamente un paciente neumónico.

En un diagrama:

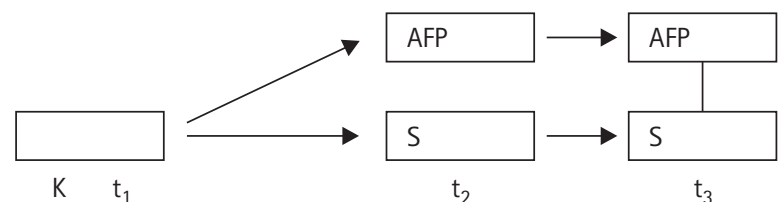

doi: 10.3989/arbor.2011.747n1002
El esquema muestra cómo $S$-signos y sintomas- y AFP -alteraciones anatomo-fisio-patológicas- evolucionan desde el comienzo a la terminación en $t_{3}$ de la enfermedad, de manera prevista por $\mathrm{M}$-crisis o muerte de $\mathrm{K}$ a la semana-. A su vez, la curación puede ser total, o con algunos rastros cicatrizales -secuelas de la enfermedad-. La neumonía, al contrario de muchas otras enfermedades no conduce generalmente a la cronicidad -otra de las posibles evoluciones-. Es innecesario remarcar que se trata de la evolución espontánea o natural de la neumonía, previa a la existencia de los antibióticos; una evolución que ahora debe entenderse como el resultado de la interacción entre los sucesos del organismo y los cambios que provoca la acción del médico introduciendo la medicación. En esta evolución dialéctica, el pronóstico es una variable dependiente de la efectividad del medicamento y corregida por éste -algo que si se cumple corrobora también el diagnóstico-. Si cura rápidamente con derivados de la penicilina o equivalentes, se trató de una neumonía.

Nuevamente insisto en que los sucesivos esquemas, expuestos en su forma más general, son aptos para describir la estructura de todo diagnóstico médico, y por consiguiente de todo caso -ejemplar- de la clínica médica.

Si ahora quisiéramos responder a qué puede entenderse por un ejemplar de la teoría clínica de las enfermedades -sabiendo que la clínica sintetiza la totalidad del conocimiento médico- podríamos hacerlo de una manera que también es similar a la respuesta de la concepción estructuralista, con la salvedad de lo que explicitaremos posteriormente.

$\mathrm{K}$-y generalizando para abarcar con nuestra estructura a todos los ejemplares de la totalidad de las enfermedades clínicas- es un ejemplar de la teoría clínica si es un ejemplar no teórico, que puede ser entendido como ejemplar teórico y que al cumplir leyes relacionales y evolutivas que relacionan entre sí a todos los elementos de la teoría, se constituye en un ejemplar actual.

"x es ejemplar de TC si es un ejemplar no teórico, luego teórico, y finalmente actual"

Avanzaré algo más en mi caracterización de las enfermedades.

ARBOR Vol. 187I 747 enero-febrero [2011] 15-24 ISSN: 0210-1963 
Diré que el término que designa a una enfermedad dada nombra el grupo -mereológico- de sus ejemplares, constituido por los casos actuales de la enfermedad. Quien los agrupa es la comunidad epistémica médica, a los efectos de nombrarlos con un mismo término en el que se sintetizan sus peculiaridades estructurales, con la consiguiente economía lingüistica que se deriva de esta maniobra generalizadora. No de otra manera procede el Dr. M cuando diagnostica a $\mathrm{K}$ como portador de una neumonía. También comprende a los casos ficcionales con los que los instructores de la comunidad relatan experiencias ejemplares fundamentalmente a los fines de la enseñanza, que $M$ suele mencionarle a su discípulo a fin de interiorizarlo en las distintas variantes de la enfermedad. Y, por supuesto, al relato ficcional que hizo este autor, al describir tanto a $\mathrm{K}$ como a $\mathrm{M}$.

Haremos notar que esta caracterización coincide con un antiguo proverbio médico que expresa que no hay enfermedades sino enfermos.

\section{LA TEORÍA COMO UN GRUPO DE EJEMPLARES}

Llegado a este punto, es hora que veamos las consecuencias de la reconstrucción léxica y estructural de la enfermedad de $\mathrm{K}$ y de su generalización a los demás casos de enfermedad. Lo hicimos mediante el procedimiento de simplificar sus elementos, eliminando de ellos las características que los particularizan, y mencionándolos con un término genérico, por ejemplo, signos y síntomas o aún más sintético $S$, en vez de soplo tubárico, matidez, fiebre, etc., o $A F P$, en vez de anatomo-fisio-patología, o de su versión más extensa que habla de exudado de tales células, con la presencia de tales microorganismos, etc.

Pudiera no ser evidente a primera vista que en nuestra caracterización prescindimos de identificar a una teoría por sus modelos -como es usual en las concepciones semánticas-, obviando los problemas que presenta su relación con los sistemas físicos que intentan explicar las ciencias fácticas, y que a nuestro entender no han sido solucionados de manera satisfactoria.

En la concepción estructuralista, se acepta que los sistemas físicos -aplicaciones de una teoría en la terminología estándar- son un subconjunto de modelos matemáticos caracterizados por los elementos no teóricos. Prima facie pareciera que un subconjunto de una entidad matemática debiera ser otra entidad matemática, no un sistema físico, y que allí hay un problema de compatibilidad ontológica y categorial, en el que se vislumbran en un nuevo contexto las añejas dificultades del platonismo $0^{4}$.

Nuestra estrategia evita estos inconvenientes, reduciendo la reconstrucción a sus ejemplares fácticos -casos-, en los que invocar su estructura no posee mayor dificultad ontológica o léxica que expresar que la estructura del cuerpo humano consta de cabeza, tronco y extremidades, definiendo estructura como un sistema en el que existen elementos y relaciones entre ellos 5 .

En esta concepción, una teoría consta únicamente de ejemplares estructuralmente caracterizados, y la palabra general que los abarca -enfermedad- no posee más contenido que el de estos ejemplares, y se refiere exclusivamente a ellos, no a una supuesta entidad abstracta llamada "enfermedad".

\section{EL USO dE UNA TEORÍA}

Bajo este epígrafe me voy a referir al mecanismo cognoscitivo que emplea $\mathrm{M}$ cuando diagnostica a $\mathrm{K}$.

En sintesis, se trata de que $\mathrm{M}$ compara a $\mathrm{K}$ con otros casos de neumonía que conoció anteriormente, y a los que $K$, en su sintomatología, se asemeja. Es por eso que $M$ piensa que tendrá lesiones orgánicas similares a las de otros casos, y que evolucionará de manera también similar.

En esta percepción de semejanzas -que lo es de las estructuras semiológicas y clínicas de $K$, y no de sus rasgos fenoménicos, tales como su peso, color de pelo, altura, largo de la nariz- es desde donde $\mathrm{M}$ infiere que $\mathrm{K}$ padece una neumonía. No necesitó ninguna ley general para deducir de ella la enfermedad de K. Por este motivo $-y$ no por una falla expositiva- es que en los libros de texto no encontramos leyes generales o más específicas acerca de las enfermedades. Basta conocer ejemplares -en realidad, cuantos más conozca mejor- para extender ese conocimiento a otros ejemplares, distinguiéndolos de los 
anteriores por los rasgos estructurales específicos que poseen.

En la reconstrucción, pudimos constatar que el axioma evolutivo cumple los requisitos de relacionar entre sí a los distintos elementos de la teoría y predecir su evolución, como lo hacen las leyes. Sin embargo, su forma difiere de los enunciados legaliformes que hablan de todos en todo tiempo y lugar -algo que desde el nominalismo es impensable-, ya que "todos" refiere a una entidad platónica. Nuevamente, la estrategia es que este axioma describe una semejanza fundamental de los ejemplares actuales, la de su comportamiento -comprobable de ejemplar en ejemplar-, y que siempre varía en algún grado. En suma, constituye un rasgo estructural más -aunque central- de la descripción de los ejemplares.

Pero no sólo se utiliza una teoría para encontrar ejemplares que puedan ser agrupados bajo la misma denominación de los que ya se conocen, como lo hace $M$, que no produce ninguna innovación científica cuando diagnostica a K. Simplemente, lo identifica como neumónico haciendo uso de las estructuras ya conocidas, en lo que llamaremos ciencia aplicada.

Pudiera suceder que aunque $\mathrm{K}$ tuviera signos y síntomas de neumonía, en las lesiones orgánicas no se encontrara presente el bacilo neumónico, ni ningún otro ya conocido. Si éste fuera el caso, el Dr. M, que es un buen investigador clínico en enfermedades infecciosas, se propondría investigar la presencia de un germen nuevo específico que produce esas lesiones. Para ello emprende una cuidadosa búsqueda mediante la cual consigue aislar un microorganismo desconocido que, cultivado e inoculado a un animal de experimentación, reproduce las lesiones que presenta $\mathrm{K}$. $\mathrm{Ha}$ encontrado una nueva enfermedad infecciosa; quizás la llame neumonía del chacarero, en homenaje a $\mathrm{K}$ que es un hombre de campo.

Se trata de un avance del conocimiento que desde Kuhn nos hemos habituado a denominar ciencia normal, esa tarea innovadora que consiste -entre otras característicasen extender el rango de aplicabilidad de las estructuras que caracterizan a una teoría dada. Para ello simplemente aplicó los procedimientos conocidos y que permitieron encontrar otras enfermedades infecciosas, comenzando por la tuberculosis, ejemplar paradigmático con el que Robert
Koch inaugura la teoría infecciosa de las enfermedades, que incluye los postulados de Koch como metodología para corroborar la causalidad del bacilo en la enfermedad, y que $\mathrm{M}$ sigue al pie de la letra en sus investigaciones.

Nuevamente, no necesitó para hacerlo más que los ejemplares de las investigaciones anteriores, y emplear procedimientos similares para obtener resultados semejantes.

Tanto la investigación aplicada como la básica normal se hicieron sin ninguna ley general -ausente en los textos de microbiología y de enfermedades infecciosas-. Su función la realiza un axioma relacional que describe la evolución del sistema formado por los elementos teóricos y no teóricos de los ejemplares, y que propone -predice- que otros ejemplares se comportarán de manera similar a éste -0 éstos- ya conocidos.

\section{Conclusiones}

Si tomamos en cuenta el lenguaje empleado en las investigaciones, la existencia de dos planos, uno semiológico, y otro anatomo-fisio-patológico -incluyendo en este caso al microbiológico-, nos permiten responder a añejas discusiones acerca del lenguaje empírico y el teórico.

El lenguaje semiológico consta de términos que no se encuentran en el lenguaje ordinario, ni es estrictamente fenoménico. Pertenece a una teoría médica en la que sus hallazgos pueden ser corroborados observacionalmente -en la terminología de Popper, sus enunciados poseen la cualidad de la observabilidad- pero esto no sucede si no se dominan las técnicas especializadas con las que se obtienen los datos, y éstos a su vez difieren de los que observaría un lego. Palabras tales como exantema, eritema, manchas de Koplick, etc., remiten a entidades observables sólo para quienes las conozcan desde la semiología. Se trata, entonces, de un lenguaje teórico con respecto al lenguaje observacional común, pero que es no teórico en las teorías clínicas. Con él se puede construir la enfermedad semiológica.

A su vez, el lenguaje de la anatomía y fisiología patológicas, así como de la microbiología, que en las teorías clínicas se utiliza para explicar los signos y síntomas de la enfermedad, es teórico con respecto al lenguaje semiológico.

ARBOR Vol. 187I 747 enero-febrero [2011] 15-24 ISSN: 0210-1963 
Como se advierte, el ser no teórico o teórico es relativo a la teoría que se tome en cuenta, y según el rol que cumpla en ella; algo que constituye uno de los hallazgos más interesante de Joseph Sneed, cuando inaugura la concepción estructuralista de las teorías.

En una vuelta de tuerca más sobre las peculiaridades de las teorias clínicas -y quizás de muchas otras teorias- se trata no tanto de la relación entre dos planos de lenguajes, sino de dos teorías, una semiológica, y anatomo-fisio-patológica y microbiológica, la otra, que constituyen en su relación la teoría clínica6.

Agreguemos que estos lenguajes, tanto el semiológico como el clínico, hablan de $\mathrm{K}-0$ cualquier otro ser humano- $\mathrm{y}_{\text {, }}$ por lo tanto, de sistemas situados espacio-temporalmente. Todo el conocimiento biomédico - de tanta trascendencia actual por la importancia de sus descubrimientos- posee esta característica, y es por lo tanto, fisicalista en este sentido, como lo queria Otto Neurath. Sin embargo, difiere de éste en que las estructuras que describen la neumonía de $\mathrm{K}$-y cualquier otra enfermedad- no son sólo no teóricas. Como vimos, incluye tanto los elementos no teóricos como los teóricos, que a su vez pueden ser corroborados independientemente. Esta última circunstancia hace que su existencia -su realismo- no sea puesto en duda, y que el instrumentalismo no sea la epistemología que más se acomode con el conocimiento médico? ${ }^{7}$.

En cuanto a los mecanismos epistémicos comprometidos en la investigación aplicada o básica de $M$, son los propios de un nominalismo de semejanzas de corte wittgensteniano. Semejanzas que no se constituyen en un nuevo universal, dado que los ejemplares difieren en algún grado en sus estructuras, y no se predica de un único ejemplar inicial sino de la totalidad de los actuales, que proporcionan la red de parecidos que $\mathrm{M}$ utiliza para investigar los ejemplares que no conoce.

En el proceso de conocimiento, se simplifica y se generaliza desde la muy detallada historia clínica realizada por $M$, hasta la estructura de los ejemplares que presentamos sin indicar signos, sintomas o alteraciones orgánicas específicos, poniendo en su lugar esos términos genéricos, e incluso letras que los sintetizan. Una descripción es- tructural de ejemplares general, apta para la descripción no sólo de las enfermedades clínicas infecciosas, sino de toda enfermedad.

Nuestra apuesta metateórica es que el esquema puede ser idóneo para exhibir la estructura de cualquier ejemplar de cualquier teoria fáctica, extendiendo el nominalismo y el fisicalismo a la totalidad del conocimiento fáctico.

Como mostráramos, en el conocimiento biomédico no hicieron falta leyes generales. Hizo sus veces el enunciado que correlaciona signos y sintomas con lesiones orgánicas en el proceso evolutivo de la enfermedad -su axioma más básico-, que sin embargo puede formularse como una ley cuya escritura tenga la forma tradicional, a la que no es necesario atribuir más referencia que las experiencias singulares de las que se origina.

Si tomamos en cuenta el lenguaje que hablan los cientifi$\cos$, no hay ningún motivo para pensar que pueda existir un lenguaje universal de la ciencia. Cada teoría posee una jerga propia, con sus propias construcciones lingüísticas, problemas, soluciones tentativas, y sus propios ejemplares -como puede verse sin ningún esfuerzo en los libros de texto habituales-.

Si ahora consideramos la reconstrucción epistemológica de sus unidades más básicas de análisis, las teorias científicas, mostramos que la mejor manera de exhibir su estructura es mediante el análisis de sus ejemplares, empleando un lenguaje nominalista y fisicalista que surge de los mismos escritos que se reconstruyen. Un nominalismo de semejanzas apto para reconstruir al menos teorias del área biológica, psicológica y social, en las que no intervienen cálculos matemáticos. Aunque no se excluye que pueda ser una herramienta útil en la reconstrucción de teorias con un fuerte componente matemático, si se lo adapta para ese fin.

La Babel de lenguajes que avizorara el neopositivismo se ha superado de una manera impensada, devolviendo su lugar al fisicalismo y al nominalismo que al decir de Neurath estuvieron en el origen de la filosofía de la ciencia contemporánea ${ }^{8}$, y que fueron relegados por un platonismo innecesario. 
1 Aunque éstas son las disciplinas básicas del plano profundo de la enfermedad, la investigación biomédica va más allá, fundamentándolas en nociones bioquímicas, de biología molecular y de genética; disciplinas cuyo lenguaje específico puede intervenir en las descripciones causales del Dr. M.

2 Utilizo actual en el mismo sentido que el de la misma palabra en inglés: existente de hecho, real.

3 Nuevamente, el sentido es de "realmente".

4 Estas dificultades surgen desde el punto de vista ontológico para quienes aceptan que las entidades matemáticas son entidades platónicas -abstractas-, de las que nunca se ha dado una respuesta satisfactoria acerca de cómo las conocemos, ni cómo se relacionan con el mundo. Si optamos por presentar el problema desde el punto de vista lingüístico -dejando de lado el ontológico-, la descripción de un sistema físico introduce nombres de individuos y especifica sus elementos, por lo que no podría ser un subconjunto de un sistema abstracto, puramente matemático, que carece de estas peculiaridades.

5 Al decir de Goodman \& Quine (1947), el nominalismo consiste -entre otras características- en negar la existencia de entidades abstractas.

6 Por si no resultara suficientemente claro, mencionaremos que la clínica médica es la disciplina que estudia y tipifica las enfermedades humanas; en este contexto llamamos teorías clínicas a cada una de las enfermedades descritas, que pueden ser simplificadas en un esquema más general que las abarque a todas. En este caso estaremos en presencia de una auténtica teoría clínica general.
7 En otras disciplinas, este realismo pudiera adoptar la forma de "interno".

8 Con respecto al nominalismo, Otto Neurath (1982, 165; traducción de C.L.) expresa: "Dado que toda la filosofía moderna tiene sus origenes en los Escolásticos, no es sorprendente que el empirismo lógico también sea la continuación de ciertos precursores escolásticos, los nominalistas". No es necesario insistir en su bien conocida posición fisicalista.

\section{BIBLIOGRAFÍA}

Balzer, Wolfgang, C. Ulises Moulines y Joseph D. Sneed (1987): An Architectonic for Science, Dordrecht: Reidel.

Bambrough, R. (1966): "Universals and Family Resemblance", en Pitcher, George (ed.) Wittgenstein, New York: Anchor Books, pp. 186-205.

Bennett, Jean Claude y Fred Plum (eds.) (1996): Cecil Textbook of Medicine, Philadelphia/London/Toronto/Montreal/Sydney/Tokio: Saunders Co.

Carnap, Rudolf (1956): "Empiricism, Semantics and Ontology", en Carnap, Rudolf, Meaning and Necessity, enlarged edition, Chicago: The University of Chicago Press, pp. 205-221.

Carnap, Rudolf (1958): "The Methodological Character of Theoretical Concepts", en Foundations of Science and the Concepts of Psychology and Psychoanalysis, Vol. I, Minnesota: Minnesota Studies in the Philosophy of Science, pp. 38-77.

Cecil, Russell L. y Robert F. Loeb (eds.) (1953): Textbook of Medicine, Philadelphia: W.B. Saunders Co.

Field, Hartry (1980): Science Without Numbers: A Defence of Nominalism, Princeton, NJ: Princeton University Press; 0xford: Blackwell. 
Goodman, Nelson y Willard van Orman Quine (1947): "Towards a Constructive Nominalism", Journal of Symbolic Logic, 12: 105-122.

Goodman, Nelson (1972): Problems and Projects, Indianapolis, New York: The Babbs Merril Company, Inc.

Hempel, Carl Gustav (1973): "The Meaning of Theoretical Therms: A Critique of the Standard Empiricist Construal", en Suppes, Patrick, Henkin, Leon, Joja, Athanese y Gr. C. Moisil (eds.) (1973), Logic, Methodology and Philosophy of Science IV, Amsterdam: North Holland, pp. 367-378.

Jaeger, Werner (1957): Paideia. Los ideales de la cultura griega, México: Fondo de Cultura Económica.

Kuhn, Thomas S. (1971): La estructura de las revoluciones científicas, México: Fondo de Cultura Económica.

Laín Entralgo, Pedro (1973): Historia universal de la medicina, Barcelona: Salvat.
Laín Entralgo, Pedro (1982): La medicina hipocrática, Madrid: Alianza Editorial.

Lorenzano, César (2002): "Una reconstrucción estructural de la bioquímica" en Díez, José Antonio y Pablo Lorenzano (eds.), Desarrollos actuales de la metateoría estructuralista: problemas y discusiones, Quilmes: Universitat Rovira i Virgili/Universidad Autónoma de Zacatecas/Universidad Nacional de Quilmes, pp. 209-231.

Neurath, Otto (1931a): "Physicalism: The philosophy of the Viennese Circle", The Monist 41: 618-623.

Neurath, Otto (1931b): "Physicalism", Scientia 50: 297-303.

Neurath, Otto (1983): Philosophical Papers 1913-1946, edited and translated by Robert S. Cohen and Marie Neurath, Vienna Circle Collection, Vol. 16, Dordrecht, Boston: Reidel.

Rodríguez-Pereyra, Gonzalo (2000): Resemblance Nominalism. A Solution to the Problem of Universals, Oxford: Oxford University Press.

Sneed, Joseph D. (1971): The Logical Structure of Mathematical Physics, Dordrecht: Reidel.

Stegmüller, Wolfgang (1973): Theorienstrukturen und Theoriendynamik, Heidelberg: Springer.

Stegmüller, Wolfgang (1979): The Structuralist View of Theories, Berlin: Springer.

Suppes, Patrick (1960): "A Comparison of the Meaning and Use of Models in Mathematics and the Empirical Sciences", en Suppes, Patrick (1969), Studies in the Methodology and Foundations of Science. Selected Papers from 1951 to 1969, Dordrecht: Reidel, pp. 10-24.

Wittgenstein, Ludwig (1958): Philosophical Investigations, Oxford: Basil Blackwell.

Wittgenstein, Ludwig (1956): Remarks on the Foundations of Mathematics, Oxford: Basil Blackwell. 